\title{
Effects of prebiotic galacto-oligosaccharide on postoperative cognitive dysfunction and neuroinflammation through targeting of the gut-brain axis
}

Xu-Dong Yang ${ }^{1+}$, Li-Kuan Wang $^{1 *+}$ (D), Hai-Yin Wu ${ }^{1,2}$ and Liang Jiao ${ }^{1,2}$

\begin{abstract}
Background: Surgery-induced neuroinflammation plays an important role in postoperative cognitive dysfunction (POCD). Gut microbiota is a key regulator of neurological inflammation. Nurturing with prebiotics is an effective microbiota manipulation that can regulate host immunity and cognition. The aim of the present study was to test whether administration of the prebiotic Bimuno ${ }^{\circledR}$ (galactooligosaccharide (B-GOS) mixture) could ameliorate POCD and attenuate surgery-induced neuroinflammation through the microbiota-brain-axis.

Methods: Adult rats undergoing abdominal surgery under isoflurane anesthesia were fed with water or prebiotic BGOS supplementation ( $15 \mathrm{~g} / \mathrm{L})$ for 3 weeks. Novel objective recognition task was employed for testing cognitive changes on postoperative day three. Expression of microglial marker Iba-1 in the hippocampus was assessed by immunohistochemical staining. Expression levels of phenotypic gene markers of activated microglia (M1: iNOS, CD68, CD32; M2: Ym1, CD206, and SOCS3) in hippocampus were determined by quantitative polymerase chain reaction (qPCR). Inflammatory cytokines in the hippocampus were assessed using enzyme-linked immunosorbent assay (ELISA). Feces were collected for microbial community analysis.

Results: Rats exhibited an impairment in novel objective recognition 3 days after surgery compared with control rats $(P<.01)$. In the hippocampus, expressions of Iba-1 and M1 markers of surgical rats were significantly upregulated. Similarly, expressions of SOCS3 and CD206 in the hippocampus were upregulated. Additionally, increasing levels of IL-6 and IL-4 were evident in the hippocampus. Administration of B-GOS significantly alleviated cognitive decline induced by surgery $(P<.01)$. B-GOS-fed rats showed a significantly downregulated activation of microglia and expressions of M1-related genes and SOCS3 and IL-6. While there was no significant difference in expressions of CD206 and Ym1 and IL-4 between the surgical and B-GOS groups. Analysis of gut microbiome found that administration of B-GOS induced a significant change beta diversity of the gut microbiome and proliferation of Bifidobacterium and other potentially anti-inflammatory microbes.
\end{abstract}

Conclusions: Administration of B-GOS has a beneficial effect on regulating neuroinflammatory and cognitive impairment in a rat model of abdominal surgery and was associated with the manipulation of gut microbiota.

Keywords: Postoperative cognitive dysfunction, Prebiotic, Neuroinflammtion, Gut-brain axis

\footnotetext{
* Correspondence: wanglikuan3@163.com

${ }^{+}$Xu Dong Yang and Li Kuan Wang contributed equally to this work.

${ }^{1}$ Department of Anesthesiology, Peking University School and Hospital of

Stomatology, \#22 Zhongguancun South Avenue, Beijing 100081, China

Full list of author information is available at the end of the article
}

(c) The Author(s). 2018 Open Access This article is distributed under the terms of the Creative Commons Attribution 4.0 International License (http://creativecommons.org/licenses/by/4.0/), which permits unrestricted use, distribution, and

reproduction in any medium, provided you give appropriate credit to the original author(s) and the source, provide a link to the Creative Commons license, and indicate if changes were made. The Creative Commons Public Domain Dedication waiver (http://creativecommons.org/publicdomain/zero/1.0/) applies to the data made available in this article, unless otherwise stated. 


\section{Background}

Postoperative cognitive dysfunction (POCD) is an adverse complication associated with anesthesia and surgery with an incidence of 11.7 to $60 \%$ [1]. It can occur in patients of all ages and following cardiac and non-cardiac surgeries, as well as after procedure sedation [2]. The mechanisms of POCD have not been elucidated fully. Preclinical and human experiments indicate that neuroinflammation plays an important part in the progression of POCD [3, 4].

Microglia are a specialized macrophage population in the central nervous system (CNS). These cells can be activated by insult factors and are the first cells to induce the neuroinflammatory response [5]. Studies have found that surgery is associated with microglial activation and inflammation-related cytokines in brain areas that are related to cognitive decline $[6,7]$. In addition, microglia are highly plastic cells that can differentiate into complex phenotypes depending on specific microenvironmental signals within the brain. Two activated phenotypes are characterized: classically-activated (M1) with destructive pro-inflammatory and cytotoxic properties; alternativelyactivated (M2) with the main function of repair, tissue remodeling (M2a), and anti-inflammation (M2b/c) [8, 9]. Therefore, microglia are a double-edged sword for neuroinflammation. Moreover, strategies based on phenotypic modulation of microglia have been found to attenuate postoperative cognitive decline in rodents $[10,11]$.

Mounting evidence from animal and human investigations indicate that the function and microenvironment of the brain are substantially influenced by gut microbes. The connection between intestinal microbes and the brain is known as the gut-brain axis [12]. Furthermore, gut microbiota is a key regulator of neuroinflammation and the activation of microglia are also regulated by gut microbiota $[13,14]$. Therefore, regulation of gut microbiota may be a potential treatment for various neurologic diseases [15]. Prebiotics are a kind of substrate that can be selectively utilized by host microorganisms, leading to stimulation of gastrointestinal microbiota and conferring a health benefit [16]. There is increasing evidence that a diet with prebiotics has beneficial effects on host immunity and the gut-brain axis $[17,18]$.

Bimuno $^{\circ}$ (galacto-oligosaccharide (B-GOS) mixture) is a widely investigated specific nondigestible galacto-oligosaccharide especially potent in selectively promoting the proliferation of Bifidobacterium [19]. Previous studies found that B-GOS led to modulation of cortical inflammatory cytokine expression and increased the expression of brain-derived-neurotrophic factor through the manipulation of gut bacteria [20]. These effects were found to ameliorate the anxiety-like behavior induced by neuroinflammation [21]. However, the effects of prebiotic administration on POCD have not been extensively explored. The aim of the current research was to investigate whether administration of B-GOS could attenuate surgery-induced cognitive dysfunction in parallel with changes in inflammatory responses in the hippocampus, activation and phenotypic transformation of microglia, and the impact of gut microbiota composition.

\section{Methods \\ Experimental animals and B-GOS supplementation}

SPF Sprague-Dawley male rats (8 months old) were obtained from Beijing Vital River Experimental Animals Co. and housed under 12:12 light:dark cycle $\left(20 \pm 2{ }^{\circ} \mathrm{C}\right.$, humidity $50 \pm 10 \%$ ) conditions. Rats were fed standard chow and water ad libitum or a B-GOS (Bimuno ${ }^{\circledR}$, Clasado Biosciences, UK) solution according to group allocations. B-GOS solution was prepared at a concentration of $15 \mathrm{~g} / \mathrm{L}$ using sterile water. All experimental procedures were conducted in accordance with animal care guidelines and approved by the Ethics Committee of the Peking University Health Science Center (LA2016320).

\section{Experimental protocol}

We first performed a pilot study to investigate the time course of postoperative cognitive impairment in the surgical model. Briefly, 12 rats were subjected to abdominal surgery under isoflurane inhaled anesthesia. Novel objective recognition test was assessed during postoperative days 3,7 , and 14 . Four naïve control rats underwent behavioral assessments together with the 3 surgical groups. Results of the pilot study indicated the novel object preference ratio was significantly decreased 3 days after surgery $(P<.01)$. However, the recognition ratio returned to normal on postoperative day 7 (Fig. 2A).

The schematic of the study is presented in Fig. 1. Thirty rats were randomly allocated to normal drinking water and non-surgery group (Control group, $\mathrm{C}$ group), normal drinking water and surgery group (Surgery group, $\mathrm{S}$ group), or B-GOS solution and surgery group (Surgery + BGOS group, SG group), 10 rats per group. Rats in the $S$ group and SG group received abdominal surgery under isoflurane inhaled anesthesia. Rats in the Control group did not receive anesthesia or surgery. Novel objective recognition of rats was determined on postoperative day 3. Rats in all groups were sacrificed after the behavior test. Brains were extracted and fixed in $4 \%$ paraformaldehyde in preparation for immunohistochemical staining. For quantitative polymerase chain reaction (qPCR) and ELISA, the hippocampi were collected and immediately frozen in liquid nitrogen, then stored at $-80^{\circ} \mathrm{C}$. Fecal samples were collected in individual sterile EP tubes and stored at $-80^{\circ} \mathrm{C}$.

\section{Surgery}

Abdominal surgery with laparotomy combined with mesenteric ischemia-reperfusion was performed based on 
B-GOS administration 3 weeks

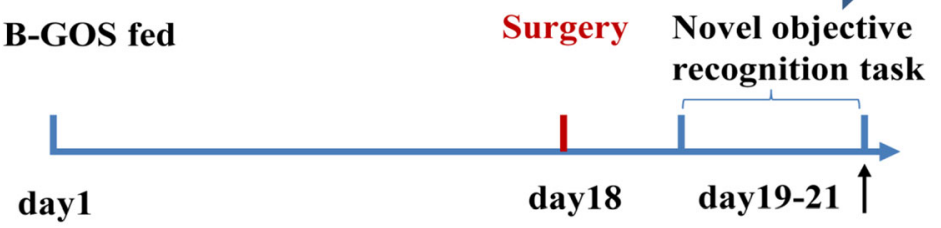

Tissue harvest

Fig. 1 Experimental schedule of study. Thirty rats received standard chow with either normal drinking water $(n=20)$ or B-GOS solution ( $n=10)$ for 21 days. On day 18, 10 water-fed rats and 10 B-GOS-fed rats received abdominal surgery under inhaled anesthesia. Novel objective recognition task was performed on days 19 to 21. Rats were sacrificed after completing the behavior test

previous reports $[22,23]$. These surgical procedures are known to result in postoperative cognitive dysfunction and neuroinflammation and are considered a model to mimic major abdominal surgery in humans [24, 25]. Briefly, rats were anesthetized under $2 \%$ isoflurane and oxygen. After the surgical area was sterilized, a vertical incision was made and the gastrointestinal tract was exteriorized and the upper mesenteric artery was reversible clamped for $30 \mathrm{~min}$. During the clamping period, the small intestine, liver, colon, and stomach were gently manipulated by a sterile probe. The muscle wall and skin were closed by sterile suture. The abdominal incision was infiltrated with $2 \%$ lidocaine solution after suturing to treat skin incision pain. Body temperature was maintained at $37 \pm 0.5^{\circ} \mathrm{C}$ with a warm blanket. All operative procedures were performed by the same team.

\section{Novel object recognition test}

Novel object recognition test is a useful tool to assess visual and spatial short-term memory for investigating the learning and memory of rodents [26]. In our experiment, rats were acclimated to the testing room and text box $(60 \mathrm{~cm} \times 60 \mathrm{~cm} \times 40 \mathrm{~cm})$ for $15 \mathrm{~min}$ on 1 day prior to exploration phase. Twenty-four hours later, rats were allowed to explore for $5 \mathrm{~min}$ two identical objects placed in the box (exploration phase). Rats that spent less than $30 \mathrm{~s}$ exploring both objects during the exploration phase were removed from further analysis. After $24 \mathrm{~h}$, one of the objects was replaced by a different object. Rats were allowed to explore these objects for $5 \mathrm{~min}$ (novel object recognition phase). Time the rats spent exploring each object was recorded with two stopwatches by two investigators blind to the animals' allocations. The novel object preference ratio was calculated as the time spent exploring the novel object, divided by the total time spent exploring the two objects.

\section{Immunohistochemical staining}

Tissue sections of the brain containing the hippocampal CA1 area were cut from paraffin-embedded brain tissues and prepared for immunohistochemical staining using the Ready-to-Use Immunohistochemistry Hypersensitivity UltraSensitive ${ }^{\mathrm{Tw}}$ S-P kit (Fuzhou Maixin Biotech, Fuzhou, China) according to the manufacturer's instructions. After deparaffinization and rehydration in graded alcohols, antigen retrieval was performed with citrate buffer for $10 \mathrm{~min}$ at $90^{\circ} \mathrm{C}$. Next, gradually cooling at room temperature, sections were washed thrice with PBS followed by addition of $3 \% \mathrm{H}_{2} \mathrm{O}_{2}$ for $10 \mathrm{~min}$ at room temperature to block endogenous peroxidase activity. The sections were then washed thrice with PBS and blocked with goat serum for $10 \mathrm{~min}$ at room temperature. After the goat serum was wiped, the sections were intubated with 1:800 rabbit-anti Iba-1(Fujifilm Wako Pure Chemical Corporation, Osaka, Japan) at $4{ }^{\circ} \mathrm{C}$ overnight, followed by intubation with biotin-labeled goat anti-rabbit secondary antibody for $10 \mathrm{~min}$ and streptomycin anti-biotin peroxidase for $10 \mathrm{~min}$ at room temperature. Next sections were labeled with 3,3'-diaminobenzidine, followed by hematoxylin counterstaining. Lastly, after washing, sections were dehydrated through gradients of ethanol and xylene. Number of Iba-1 positive cells was calculated per three random microscopic fields by two investigators blind to the allocation. Three brain slices per animal were chosen for analyses.

\section{Quantitative PCR analysis for microglia phenotypic genes}

Total RNA was extracted from the whole hippocampus using Trizol reagent (Thermo Fisher Invitrogen Life Technologies, Waltham, MA, USA). Isolated RNA was reverse transcribed into cDNA with RevertAid First Strand cDNA Synthesis Kit (Thermo Fisher). Quantitative polymerase chain reaction (qPCR) was performed using designed primers (Table 1 ) and $\mathrm{SYBR}^{\circ}$ Green Master (Roche Bioscience, Indianopolis, IN, USA) for $95^{\circ} \mathrm{C}$ for $10 \mathrm{~min}$ and $40 \mathrm{cycles}$, then at $95^{\circ} \mathrm{C}$ for $15 \mathrm{~s}$ and $60^{\circ} \mathrm{C}$ for $60 \mathrm{~s}$. Relative gene expression was calculated relative to the expression of glyceraldehyde-3-phosphate dehydrogenase (GAPDH) mRNA. 
Table 1 Gene sequences of primers

\begin{tabular}{lll}
\hline Gene & Forward sequence & Reverse sequence \\
\hline iNOS & TTCACGACACCCTTCACCAC & AGCTGGTAGGTTCCTGTTGTTTC \\
CD206 & TGTTTTGGCTGGGACTGACCTA & CGGGTGTAGGCTCGGGTAGTAG \\
CD32 & CAAGAGCCCAAATCCAGCAGT & TTGAGATAGACCAAGGATACCAGG \\
CD68 & CTGTATTGAACCCGAACAAAACC & GAGAATGTCCACTGTGCTGCTT \\
SOCS3 & GCCACTTCTTCACACTGAGCGT & GAAGGTTCCGTCGGTGGTAAAG \\
Ym1 & TCTGAATGATGGAGCCACTGATCT & GTCCTTGAGCCACTGAGCCTTA \\
GAPDH & TGGAGTCTACTGGCGTCTT & TGTCATATTTCTCGTGGTTCA \\
\hline
\end{tabular}

\section{ELISA assays}

The same weighted hippocampal tissues were homogenized in $4{ }^{\circ} \mathrm{C}$ normal saline $(1,9 \mathrm{~m} / \mathrm{v})$ and centrifuged at $3500 \mathrm{rpm}$ for $15 \mathrm{~min}$ to obtain sample supernatants. The levels of hippocampal IL-6 and IL-4 were detected using the Rat IL-6 ELISA-kit and Rat IL-4 ELISA-kit (MultiSciences, Hangzhou, China) according to the manufacturer's instructions.

\section{Absolute quantification of Bifidobacterium \\ qPCR was performed to examine the fecal Bifidobacter- ium of rats. DNA was extracted from feces using a commercially-available stool DNA kit (TIANGEN, Beijing, China) according to the manufacturer's instruction. The PCR reactions were performed in a volume of $20 \mu \mathrm{l}$ con- taining $10 \mu \mathrm{l}$ SYBR Green PCR Master Mix (Takara Bio USA, Mountain View, CA, USA), $1 \mu \mathrm{l}$ of $10 \mu \mathrm{M}$ primers, and $2 \mu \mathrm{l}$ of cDNA. qPCR was performed using the follow- ing parameters: $95^{\circ} \mathrm{C}$ for $30 \mathrm{~s}$ and 40 cycles with $95^{\circ} \mathrm{C}$ for $5 \mathrm{~s}$ and $60^{\circ} \mathrm{C}$ for $40 \mathrm{~s}$. The primer used for the quantifica- tion of Bifidobacterium was $\mathrm{F}\left(5^{\prime}\right.$-GATTCTGGCTCAGG $\left.A T G A A C G C-3^{\prime}\right)$ and $\mathrm{R}\left(5^{\prime}-C T G A T A G G A C G C G A C C C C A\right.$ $\left.T-3^{\prime}\right)$. The gene copy numbers were quantified by com- paring standard serial dilutions from $10^{1}$ to $10^{5}$ copies of plasmid DNA. Results were expressed as copies of DNA per gram of stool and Log10 transformed for statistical analysis.}

\section{High-throughput analysis of fecal microbial community}

DNA from feces was extracted using a commercially-available stool DNA kit (Omega Bio-Tek, Norcross, GA, USA) following the manufacturer's instructions. Purity and quality of the genomic DNA were checked on $0.8 \%$ agarose gels. Amplification of $16 \mathrm{~S}$ rRNA gene was performed with the primers $357 \mathrm{~F}(A C T C C T A C G G G A G G C A G C A G)$ and 806R(GGACTACHVGGGTWTCTAAT), which target the V3-V4 hypervariable region. All samples were deep sequenced on a Miseq platform using the Illumina Analysis Pipeline version 2.6 (Illumina, San Diego, CA, USA). After screening the raw data by excluding low-quality reads, such as sequences shorter than $200 \mathrm{bps}$, low-quality scores ( $\leq$ 20 ), contained ambiguous bases, or did not exactly match primer sequences and barcode tags, the high-quality reads were separated using sample-specific barcode sequences and trimmed with Illumina Analysis Pipeline Version 2.6. Next, the dataset was analyzed using QIIME (http://qiime.org), the online bioinformatics pipeline that performs microbiome analysis from raw DNA sequencing data. The sequences at a similarity level of $97 \%$ were clustered into operational taxonomic units (OTUs), to generate rarefaction curves and to calculate the richness and diversity indices. All sequences were classified into different taxonomic groups using the Ribosomal Database Project Classifier tool. To evaluate $\alpha$ diversity of the gut microbiome, chao 1 index was calculated. Principal coordinate analyses (PCoA) based on the unweighted UniFrac distances was performed in QIIME to examine the similarity between different samples and determined using PERMANOVA analysis.

\section{Statistics}

Data were expressed as the mean and standard error. For multiple comparisons, a one-way ANOVA followed by posthoc Tukey test were performed. The Kruskal-Wallis and Dunn's tests were used for nonparametric comparison. $P<.05$ was regarded statistically significant. Statistical analysis was performed with Graphpad Prism software (GraphPad Software, La Jolla, CA, USA).

\section{Results}

\section{Cognitive changes after surgery}

Two rats (1 in S group and 1 in SG group) were excluded due to insufficient time spent on total objects during the exploration phase. The time spent on total objects in exploration phase did not differ significantly between groups (Fig. 2B). Novel object recognition index was significantly decreased 3 days after abdominal surgery $(P<.01)$. Rats in SG group showed significantly improved cognition compared with Surgery group rats $(P$ $<.01$ ) (Fig. 2C).

\section{Iba-1 expression in the CA1 area of the hippocampus}

Results from immunohistochemical staining indicated that the Iba-1 positive cell was significantly increased in the CA1 area of the hippocampi of rats in the Surgery group $(P<.01)$. B-GOS-fed rats show a reduced Iba-1 positive cell in the hippocampal CA1 area after surgery $(P<.01)$ (Fig. 3).

\section{Expressions of phenotypic-related genes of activated microglia in hippocampus}

Activated microglia are commonly characterized by their signature genes to distinguish between different active phenotypes. In our study, phenotype genes were investigated based on qPCR results. We found that the expression levels of M1-type genes (iNOS, CD68, CD32) in hippocampus were significantly elevated after surgery. 

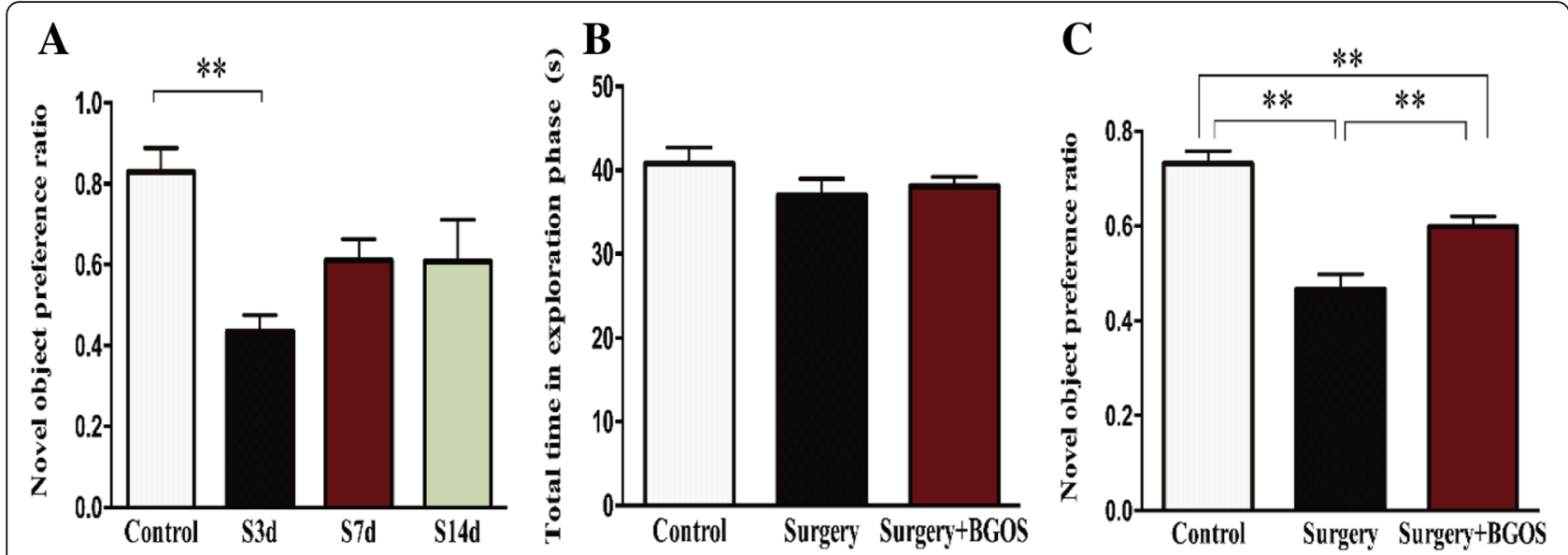

Fig. 2 Time course of POCD and effects of B-GOS on surgery-induced impairment of novel objective recognition. Data are presented as mean \pm SEM. (a) Time course of novel objective recognition following surgery. (b) Total exploration time of total objects during exploration phase. (c) Novel object preference ratio of recognition phase. S3d, S7d, and S14d = rats that underwent behavioral testing during days 3, 7, and 14 after surgery. ${ }^{* *} P<.01$ for comparisons shown

Feeding with B-GOS effectively inhibited the increased M1-type gene levels in the hippocampus induced by surgery. However, we observed different trends in expressions of M2-typical genes (CD206, SOCS3, Ym1). Expressions of CD206 and SOCS3 in the surgery group were elevated while Ym1 was reduced. B-GOS downregulated the expression of SOCS3. But, changes in expressions of CD206 and Ym1 induced by surgery were not significantly impacted by B-GOS (Fig. 4).

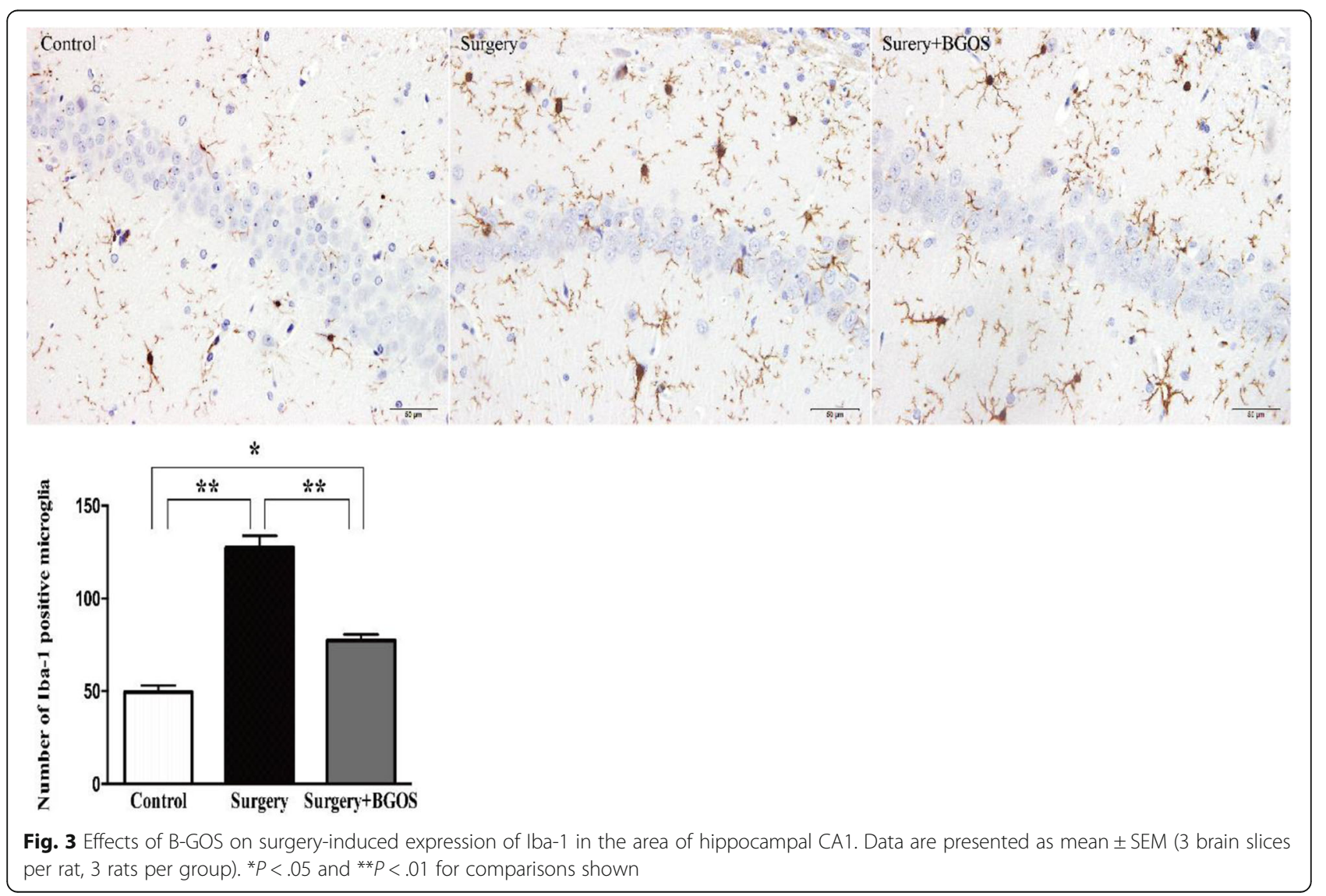


Expressions of inflammatory cytokines in hippocampus Protein expressions of IL- 6 and IL-4 in rat hippocampus were measured. Hippocampal levels of IL-6 and IL-4 was significantly higher in the surgery group compared with the control group $(P<.01)$. B-GOS consumption downregulated the level of IL-6 in hippocampus induced by surgery $(P<.01)$. While surgery-induced upregulation of IL-4 was not significantly affected by administration of B-GOS (Fig. 5).

\section{Microbial community analysis}

qPCR results showed a higher concentration of Bifidobacterium in the B-GOS groups than in animals in the control and surgery groups $(P<.01)$ (Fig. 6A). Taxonomy of fecal microbiota was assessed to observe alterations in fecal microbiota using high-throughput analysis of $16 \mathrm{~S}$ RNA. There was no significant difference between the three groups in the values of Chaol index (Fig. 6B). Beta diversity was calculated and principal coordinate analysis was performed. Fecal microbiota from B-GOS-fed rats and normal drinking water-fed rats could be significantly separated by principal coordinate analysis (Fig. 6C). Further, we compared fecal microbiota composition between groups at multiple taxonomic levels. At the phylum level, the relative abundance of Actinobacteria was significantly increased in B-GOS-fed rats. At family level, Lactobacillaceae and Lachnospiraceae were significantly more abundant in the fecal microbiota of rats fed with B-GOS than in those given normal drinking water, while Ruminococcaceae was less abundant in the B-GOS group (Fig. 7).

\section{Discussion}

In the present study, we found that: (1) abdominal surgery-induced decline in objective recognition was partially alleviated by administering B-GOS to rats; (2) B-GOS suppressed microglial overactivation and decreased the proportion of M1 phenotypic-microglia induced by surgery; (3) feeding with B-GOS exerted an adequate prebiotic role in promoting proliferation of potentially anti-inflammatory microbes, which may have contributed to regulation of the neuroinflammatory response induced by surgery through the microbiota-brain-axis.

The process of anesthesia and surgery is a strong and acute inflammatory initiator. It triggers a systemic inflammatory response and provokes release of circulatory inflammatory mediators [27]. Overexpression of these inflammatory cytokines disrupts the blood-brain barrier and induces microglial activation and a neuroinflammatory

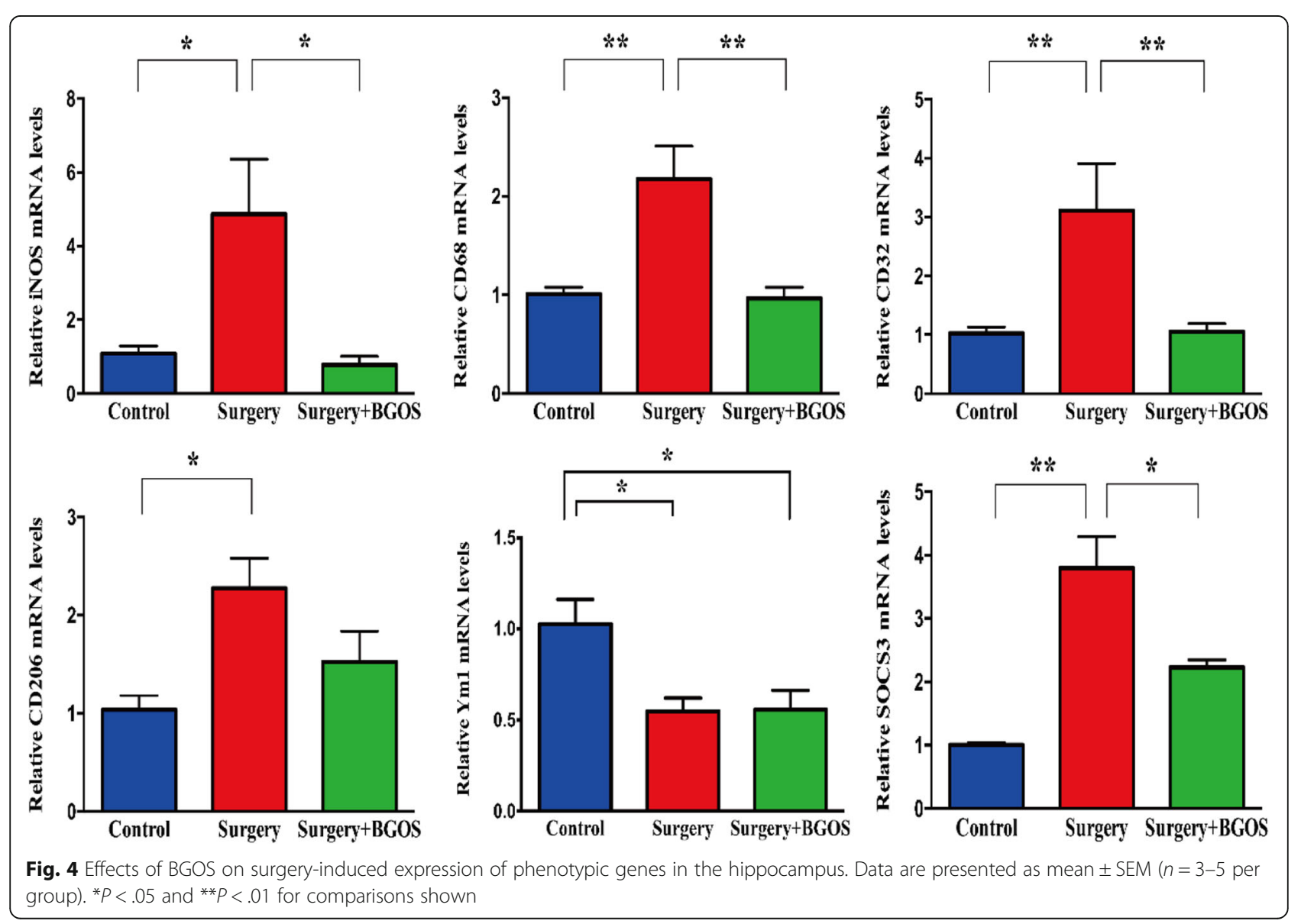



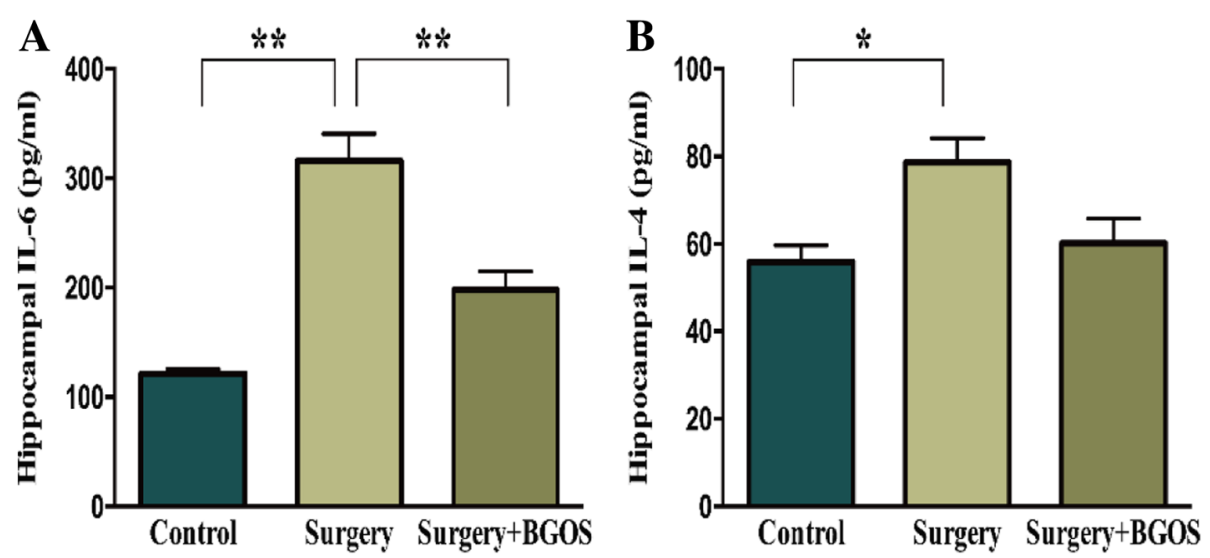

Fig. 5 Effects of B-GOS on surgery-induced inflammatory cytokines in the hippocampus. Concentrations of IL-6 (a) and IL-4 (b) in hippocampus. Data are presented as mean \pm SEM $\left(n=3\right.$ per group). ${ }^{*} P<.05$ and ${ }^{* *} P<.01$ for comparisons shown

reaction $[28,29]$. Exaggerated neuroinflammation is associated with damage of synapses and neurons, and leads to POCD [4]. In this process, activation of microglia plays a major role in neuroinflammation. Increasing evidence indicates that POCD is associated with over-activation of microglia [30]. The hippocampus is the most vulnerable region during the neuroinflammatory response [6]. Consistent with previous studies, an increased expression of Iba-1 and pro-inflammatory cytokines in the hippocampus paralleled by cognitive decline after surgery were observed in the present study.

Main aims of our study were to clarify whether inhibition of surgery-induced neuroinflammation by administering prebiotics is a potential treatment for POCD. Gut microbiota are important for healthy function of the immune and endocrine systems, and can affect various subsets of immune cells. Thus, regulation of the microbiota can impact the host inflammatory response [31]. For the brain, increasing attention is being paid to how the gut microbiota impacts behavior and neuroinflammation.
Studies in germ-free mice have shown that the microbiota regulates the blood-brain-barrier and is essential for hippocampal neurogenesis [32]. Erny et al. found that the gut microbiota is requisite for maintaining the steady-state conditions of microglia. In their study, both germ-free and antibiotic-treated mice showed impaired microglial function. Moreover, expressions of some phenotypic genes were changed in the absence of microbiota [33]. Based on the growing findings, strategies are emerging for shifting the composition of gut microbiota via non-dietary or dietary interventions to manipulate neuroinflammation in neuropsychologic disease. The immunoregulatory effect of prebiotics has been proven in previous studies. Savignac et al. revealed that mice fed with prebiotics prevented LPS-induced anxiety and normalized the elevation of IL-1beta level in the brain [21]. Other studies have shown a beneficial role of prebiotic treatment for stress- and beta-amyloid-induced neuroinflammation and cognitive impairment [34, 35]. Therefore, investigations of microbiotabased interventions to resolve surgery-induced

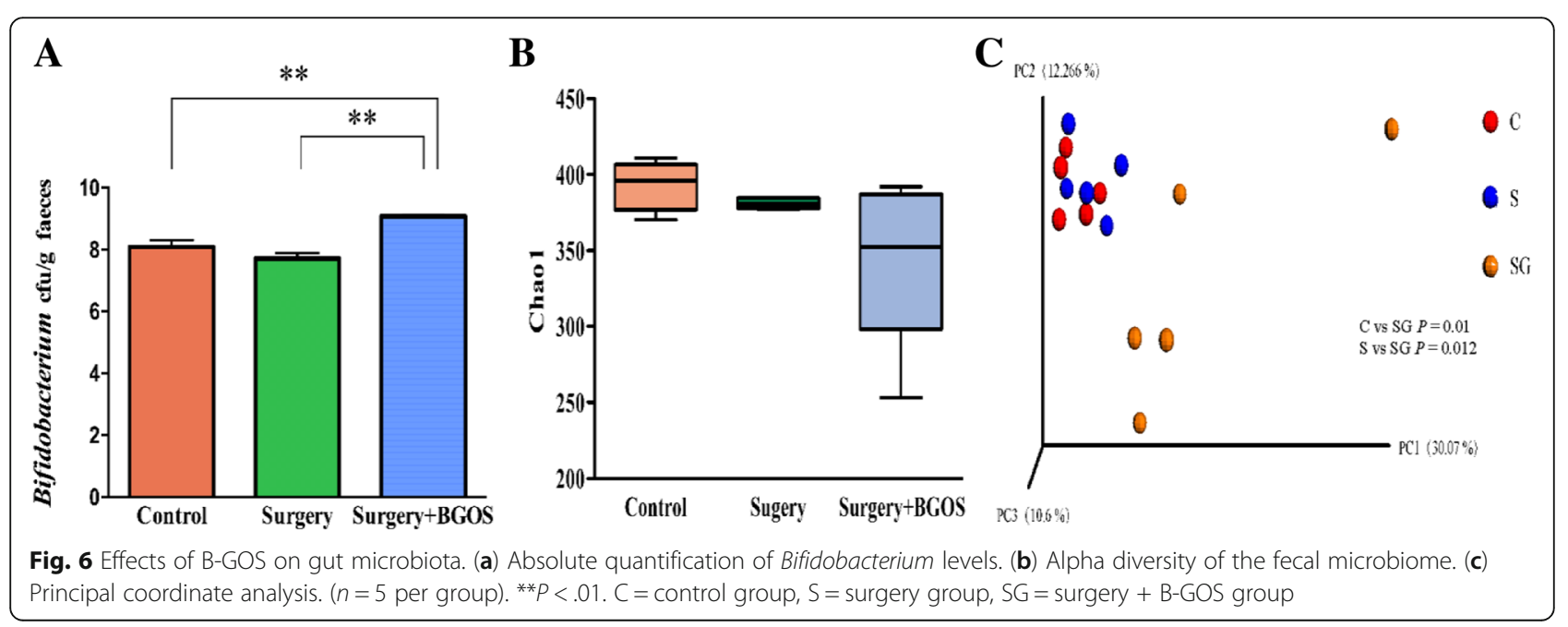



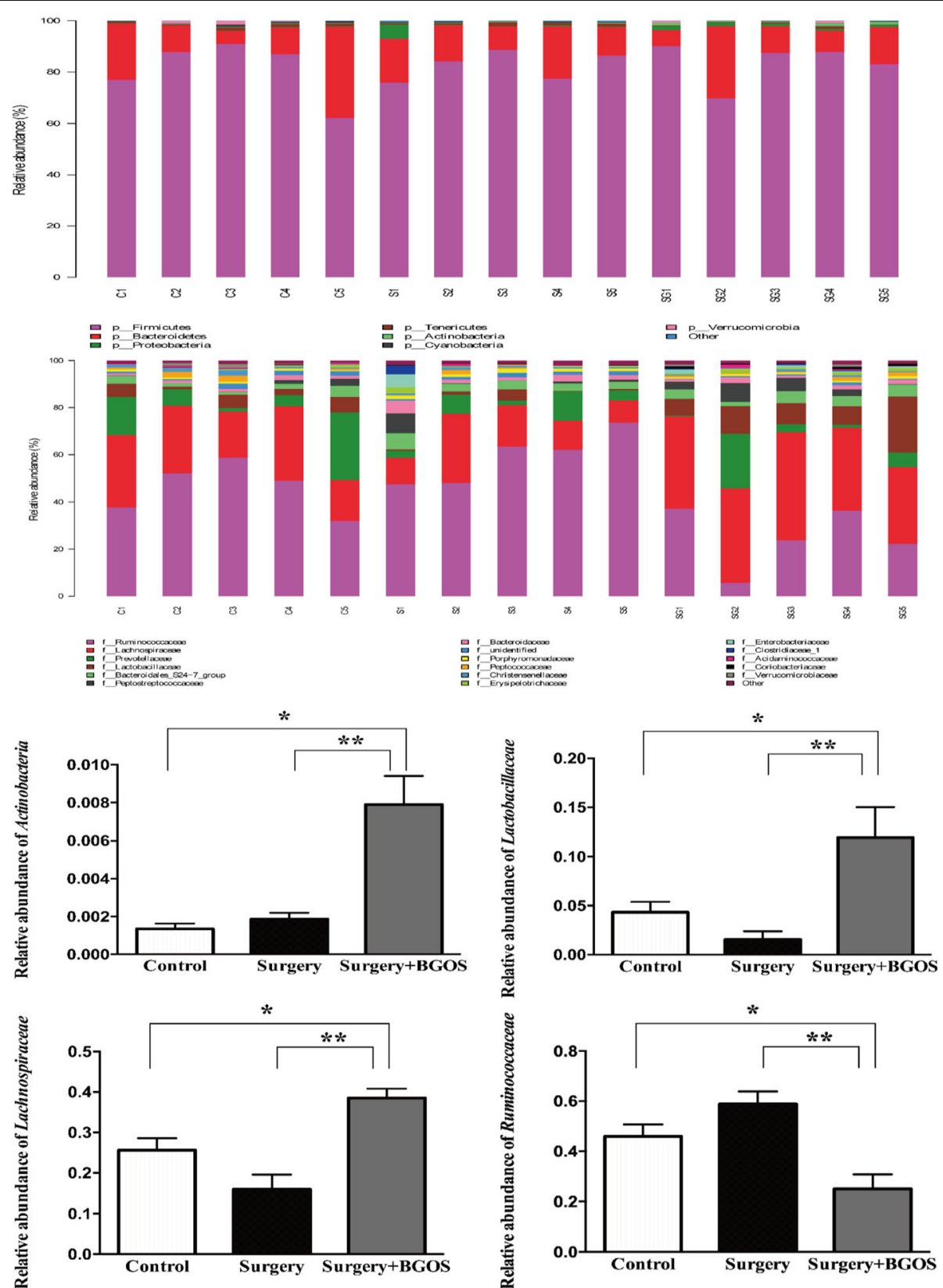

Fig. 7 Classification and relative abundance of fecal microorganism at phylum and family levels. ( $n=5$ per group). ${ }^{*} P<.05$, ${ }^{* *} P<.01$. $C=$ control group, $\mathrm{S}=$ surgery group, SG = surgery + B-GOS group

neuroinflammation and cognitive dysfunction are warranted. In the present study, we found that the prebiotic B-GOS attenuated surgery-induced cognitive dysfunction and overactivation of microglia.

The presence of multiple activation phenotypes for microglia is an important concept in understanding the role of microglia in inflammation. These different activated states can be characterized and identified by cellular surface receptors or specific gene markers. M1 phenotypic microglia are injurious and detrimental to neurologic outcomes as shown in many models of neurologic diseases, including POCD $[8,36]$. In agreement with previous studies, our investigation found that trauma from surgery induced an increased expression of M1-related markers in the hippocampus, and treatment with B-GOS significantly attenuated these increased levels of M1-related markers in the hippocampus following surgery. Production of inflammatory related cytokines was also investigated to identify the M1/M2 phenotypic microglia from the viewpoint of functions. IL-6 has been considered an important pro-inflammatory cytokine in pathogenesis of POCD [37]. Furthermore, IL-6 is thought to be secreted specifically by 
M1 microglia [38]. Consistent with the profile of M1-related gene markers, the present study found B-GOS feeding could depress the elevation of IL- 6 induced by surgery. Therefore, these results suggest that consumption of B-GOS appears to attenuate activation of M1subpopulations of microglia in surgery-induced neuroinflammatory response.

We also explored the expression of M2-typical genes and cytokines. SOCS3 is a gene marker of M2-immunomodulatory $(\mathrm{M} 2 \mathrm{~b} / \mathrm{c})$ microglia, which is thought to be a key negative regulator of IL-6 signaling [39]. A previous in vitro study found that pro-inflammatory stimuli induced M1 markers in parallel with the M2-immunomodulatory markers included SOCS3 [40]. Our study also indicated changes in expression of SOCS3 paralleled by the expression of M1-related markers and levels of IL-6. Ym1 and CD206 have been widely investigated M2-typical markers that are involved in tissue remodeling and enhancement of phagocytosis (M2a) [9]. In our study, post-surgical expression of $\mathrm{CD} 206$ was elevated while the expression of Ym1 was reduced. However, changes in expression of CD206 and Ym1 induced by surgery were not significantly impacted by B-GOS. IL-4 is a M2a-related microglial cytokine and has been found to play a beneficial role in surgery-induced neuroinflammation and cognitive dysfunction [41]. In the present study, however, surgery-induced increased expression of IL- 4 was not affected by administration of B-GOS. Taken together, these findings show that inhibition of M1 activation by B-GOS is mainly involved in the improvement of cognition after surgery while the effect of B-GOS on M2 phenotypic microglia needs more study to clarify the role of prebiotics on microglial phenotypes in POCD as well as on other neuroinflammatory diseases.

To further elucidate the underlying mechanism of B-GOS on POCD and neuroinflammation targeting the brain-gut axis, we collected the feces of rats for microbial community analysis. Bifidobacterium is an "immunobiotic" that can beneficially regulate the neuroinflammatory response and behavior in many models of neuroinflammatory-related diseases $[42,43]$. The properties of B-GOS have been consistently shown to selectively increase Bifidobacterium in both humans and animals [44-46]. This bifidogenic action of B-GOS was enhanced in our study. PCR analysis revealed a significant growth of Bifidobacterium after administration of B-GOS. Further, we performed high-throughput analysis of 16S RNA in order to investigate the diversity and bacterial community structure of rats. Alpha diversity was applied to analyze the richness of microbial taxa in a sample. Beta diversity was used to measure the magnitude of differences in community composition among the samples. We did not find significant differences in Chaol index. This suggests that administration of B-GOS did not affect species richness of the fecal microbiota in rats. However, PCoA results indicated that gut microbiota of B-GOS fed rats were significantly distinct from the other two groups. These results suggest that the effects of prebiotic B-GOS are attributed to overall regulation of intestinal flora rather than to stimulation of a specific type of microorganism. In addition, based on high-throughput analysis, OTU abundance and taxonomic profiles indicated a significantly increased abundance of Actinobacteria, Lactobacillus, and Lachnospiraceae and a decrease in abundance of Ruminococcus. Lactobacillus is a widely studied microbial strain that has beneficial effects on neuroinflammatory-related disease. Lactobacillus potently protects memory deficit and neuroinflammation in mouse model of aging and Alzheimer disease [47, 48]. Lactobacillus consumption has been found to restore cognition in obese-insulin resistant rats and decrease overactivation of microglia in the hippocampus [17]. Studies have found that Lachnospiraceae play an essential role in regulation of gut immune homeostasis. Lachnospiraceae depletion was found in the rodent model of inflammation. Furthermore, Lachnospiraceae showed negative correlation with place recognition memory in the rat model of high-energy diet-induced cognitive impairment [49]. Burokas et al. found that combined administration of fructooligosaccharides (FOS) and GOS induced an increase in relative abundance of Lactobacillus and a decrease in relative abundance of Ruminococcus. These prebiotic effects of FOS and GOS could inhibit stress-induced hippocampal inflammation and attenuated stress-related behaviors across domains relevant to anxiety, depression, and cognition [34]. Another investigation also demonstrated that treatment with prebiotic FOS from the medicinal plant Morinda officinalis can promote an increase in Lactobacillus population and reduce the population of Ruminococcus and ameliorate the memory deficiencies and neuroinflammation induced by $A \beta$ through targeting of the microbiota-brain axis [35]. These findings support the notion that the positive effects on the beneficial microbes of prebiotics have a positive role in the neuroinflammatory response and behavior impairments. To our knowledge, the present study is the first to demonstrate that the use of prebiotics to modulate the gut composition of microbiota has beneficial effects on surgery-induced neuroinflammatory response and cognitive dysfunction.

There are several limitations of the present study that must be considered. First, we did not establish a separate animal group exposed to anesthesia alone. Although previous studies have suggested that inhaled anesthetics may induce impairment in learning and memory, other studies have shown that the anesthetic method employed in our study was not associated with any adverse effects on cognition and neuroinflammatory response $[27,50,51]$. Second, we evaluated cognitive function in rats at a single time point rather than a period that includes complete 
surgical recovery. This test point was selected based on the results of our small-sized pilot experiment. Furthermore, similar results from other studies using a similar surgery model to observe POCD found that cognitive dysfunction was most pronounced within the first week after surgery [6]. Third, we only used the novel objective task as a tool to evaluate the changes of postoperative cognition. This behavior test does not require any external motivation, reward, or punishment and does not generate stressful conditions. In addition, delay of the time interval between the exploration and the recognition phase is more suitable to detect the hippocampus-dependent cognition [52]. However, many other brain regions are involved in the formation and processing of learning and memory, including the cortex and amygdala [53, 54]. Impairment of these brain areas is associated with changes in cognition in models of POCD $[7,50]$. Thus, it is necessary to perform a more well-designed protocol and appropriate behavior test to completely evaluate the cognitive impairment and effects of prebiotics in POCD.

\section{Conclusions}

In summary, our data provides laboratory evidence for the beneficial role of the prebiotic B-GOS on surgery-induced neuroinflammation and cognitive dysfunction in early postsurgery through the gut-brain axis. More important, this study supports the potential for a new therapy for POCD in the field of nutritional neuropsychopharmacology.

\section{Abbreviations}

B-GOS: Bimuno ${ }^{\oplus}$ galacto-oligosaccharide; CNS: central nervous system; ELISA: enzyme-linked immunosorbent assay; iNOS: inducible nitric oxide synthase; OTUs: operational taxonomic units; PBS: phosphate buffer solution; PCOA: principal coordinate analysis; POCD: postoperative cognitive dysfunction; qPCR: quantitative polymerase chain reaction;

SOCS3: suppressor of cytokine signaling 3; Ym1: chitinase-3 like protein

\section{Acknowledgements}

The authors thank Dr. Miao Liu for her kind technical assistance with this project. We are also grateful to Nissi S. Wang, MSc, for reviewing and editing the manuscript.

\section{Funding}

The present study was supported by the Science Foundation for Youth Scholar of Peking University School and Hospital of Stomatology (No. PKUSS20160109).

\section{Availability of data and materials}

The datasets used and/or analysed during the current study are available from the corresponding author upon reasonable request.

\section{Authors' contributions \\ L-KW and X-DY designed the project; X-DY, L-KW, and H-YW performed the experiments and data collection; $\mathrm{L} J$ was responsible for animal allocation and data analysis; X-DY and L-KW prepared the figures and drafted the manuscript. All authors read and approved the final manuscript.}

\section{Ethics approval and consent to participate}

All experimental procedures were approved by The Ethics Committee of the Peking University Health Science Center (LA2016320).
Consent for publication

Not applicable.

\section{Competing interests}

None of the authors has a conflict of interest to declare.

\section{Publisher's Note}

Springer Nature remains neutral with regard to jurisdictional claims in published maps and institutional affiliations.

\section{Author details \\ ${ }^{1}$ Department of Anesthesiology, Peking University School and Hospital of Stomatology, \#22 Zhongguancun South Avenue, Beijing 100081, China. ${ }^{2}$ National Engineering Laboratory for Digital and Material Technology of Stomatology, Beijing Key Laboratory of Digital Stomatology, \#22 \\ Zhongguancun South Avenue, Beijing 100081, China.}

Received: 23 May 2018 Accepted: 16 November 2018

Published online: 30 November 2018

\section{References}

1. Needham MJ, Webb CE, Bryden DC. Postoperative cognitive dysfunction and dementia: what we need to know and do. Br J Anaesth. 2017;119:11 15-i25.

2. Evered L, Scott DA, Silbert B, Maruff P. Postoperative cognitive dysfunction is independent of type of surgery and anesthetic. Anesth Analg. 2011;112: 1179-85.

3. Krenk L, Rasmussen LS, Kehlet H. New insights into the pathophysiology of postoperative cognitive dysfunction. Acta Anaesthesiol Scand. 2010;54:951-6.

4. Riedel B, Browne K, Silbert B. Cerebral protection: inflammation, endothelial dysfunction, and postoperative cognitive dysfunction. Curr Opin Anaesthesiol. 2014;27:89-97.

5. Dheen ST, Kaur C, Ling EA. Microglial activation and its implications in the brain diseases. Curr Med Chem. 2007;14:1189-97.

6. Hovens IB, Schoemaker RG, van der Zee EA, Absalom AR, Heineman E, van Leeuwen BL. Postoperative cognitive dysfunction: involvement of neuroinflammation and neuronal functioning. Brain Behav Immun. 2014; 38:202-10.

7. Hovens IB, van Leeuwen BL, Nyakas C, Heineman E, van der Zee EA, Schoemaker RG. Postoperative cognitive dysfunction and microglial activation in associated brain regions in old rats. Neurobiol Learn Mem. 2015;118:74-9.

8. Eggen BJ, Raj D, Hanisch UK, Boddeke HW. Microglial phenotype and adaptation. J Neurolmmune Pharmacol. 2013;8:807-23.

9. Hu X, Leak RK, Shi Y, Suenaga J, Gao Y, Zheng P, et al. Microglial and macrophage polarization-new prospects for brain repair. Nat Rev Neurol. 2015;11:56-64.

10. Lee JH, Kam EH, Kim SY, Cheon SY, Kim EJ, Chung S, et al. Erythropoietin attenuates postoperative cognitive dysfunction by shifting macrophage activation toward the M2 phenotype. Front Pharmacol. 2017;8:839.

11. Wang HL, Liu H, Xue ZG, Liao QW, Fang H. Minocycline attenuates postoperative cognitive impairment in aged mice by inhibiting microglia activation. J Cell Mol Med. 2016;20:1632-9.

12. Smith PA. The tantalizing links between gut microbes and the brain. Nature. 2015;526:312-4.

13. Erny D, Prinz M. Microbiology: gut microbes augment neurodegeneration. Nature. 2017;544:304-5.

14. Kamada N, Seo SU, Chen GY, Nunez G. Role of the gut microbiota in immunity and inflammatory disease. Nat Rev Immunol. 2013;13:321-35.

15. Tremlett H, Bauer KC, Appel-Cresswell S, Finlay BB, Waubant E. The gut microbiome in human neurological disease: a review. Ann Neurol. 2017; 81:369-82.

16. Gibson GR, Hutkins R, Sanders ME, Prescott SL, Reimer RA, Salminen SJ, et al. Expert consensus document: the international scientific Association for Probiotics and Prebiotics (ISAPP) consensus statement on the definition and scope of prebiotics. Nat Rev Gastroenterol Hepatol. 2017;14:491-502.

17. Chunchai T, Thunapong W, Yasom S, Wanchai K, Eaimworawuthikul S, Metzler $G$, et al. Decreased microglial activation through gut-brain axis by prebiotics, probiotics, or synbiotics effectively restored cognitive function in obese-insulin resistant rats. J Neuroinflammation. 2018;15:11. 
18. Pistollato F, Sumalla Cano S, Elio I, Masias Vergara M, Giampieri F, Battino M. Role of gut microbiota and nutrients in amyloid formation and pathogenesis of Alzheimer disease. Nutr Rev. 2016;74:624-34.

19. Vulevic J, Drakoularakou A, Yagoob P, Tzortzis G, Gibson GR. Modulation of the fecal microflora profile and immune function by a novel transgalactooligosaccharide mixture (B-GOS) in healthy elderly volunteers. Am J Clin Nutr. 2008;88:1438-46.

20. Williams S, Chen L, Savignac HM, Tzortzis G, Anthony DC, Burnet PW. Neonatal prebiotic (BGOS) supplementation increases the levels of synaptophysin, GluN2A-subunits and BDNF proteins in the adult rat hippocampus. Synapse. 2016;70:121-4.

21. Savignac HM, Couch Y, Stratford M, Bannerman DM, Tzortzis G, Anthony DC, et al. Prebiotic administration normalizes lipopolysaccharide (LPS)-induced anxiety and cortical 5-HT2A receptor and IL 1-beta levels in male mice. Brain Behav Immun. 2016:52:120-31.

22. Hovens IB, Schoemaker RG, van der Zee EA, Heineman E, Nyakas C, van Leeuwen BL. Surgery-induced behavioral changes in aged rats. Exp Gerontol. 2013;48:1204-11.

23. Rosczyk HA, Sparkman NL, Johnson RW. Neuroinflammation and cognitive function in aged mice following minor surgery. Exp Gerontol. 2008;43:840-6.

24. Hovens IB, van Leeuwen BL, Mariani MA, Kraneveld AD, Schoemaker RG. Postoperative cognitive dysfunction and neuroinflammation; cardiac surgery and abdominal surgery are not the same. Brain Behav Immun. 2016;54:178-93.

25. Tian XS, Tong YW, Li ZQ, Li LX, Zhang T, Ren TY, et al. Surgical stress induces brain-derived neurotrophic factor reduction and postoperative cognitive dysfunction via glucocorticoid receptor phosphorylation in aged mice. CNS Neurosci Ther. 2015;21:398-409.

26. Savage S, Ma DIII. Animal behaviour testing: memory. Br J Anaesth. 2014; 113:6-9.

27. Cibelli M, Fidalgo AR, Terrando N, Ma D, Monaco C, Feldmann M, et al. Role of interleukin-1 beta in postoperative cognitive dysfunction. Ann Neurol. 2010;68:360-8.

28. Terrando N, Eriksson LI, Ryu JK, Yang T, Monaco C, Feldmann M, et al. Resolving postoperative neuroinflammation and cognitive decline. Ann Neurol. 2011;70:986-95.

29. Wan Y, Xu J, Ma D, Zeng Y, Cibelli M, Maze M. Postoperative impairment of cognitive function in rats: a possible role for cytokine-mediated inflammation in the hippocampus. Anesthesiology. 2007;106:436-43.

30. Hovens IB, Schoemaker RG, van der Zee EA, Heineman E, Izaks GJ, van Leeuwen BL. Thinking through postoperative cognitive dysfunction: how to bridge the gap between clinical and pre-clinical perspectives. Brain Behav Immun. 2012;26:1169-79.

31. Dorrestein PC, Mazmanian SK, Knight R. Finding the missing links among metabolites, microbes, and the host. Immunity. 2014;40:824-32.

32. Braniste V, Al-Asmakh M, Kowal C, Anuar F, Abbaspour A, Toth M, et al. The gut microbiota influences blood-brain barrier permeability in mice. Sci Transl Med. 2014;6:263ra158.

33. Erny D, Hrabe de Angelis AL, Jaitin D, Wieghofer P, Staszewski O, David E, et al. Host microbiota constantly control maturation and function of microglia in the CNS. Nat Neurosci. 2015;18:965-77.

34. Burokas A, Arboleya S, Moloney RD, Peterson VL, Murphy K, Clarke G, et al. Targeting the microbiota-gut-brain Axis: prebiotics have anxiolytic and antidepressant-like effects and reverse the impact of chronic stress in mice. Biol Psychiatry. 2017;82:472-87.

35. Chen D, Yang X, Yang J, Lai G, Yong T, Tang X, et al. Prebiotic effect of Fructooligosaccharides from Morinda officinalis on Alzheimer's disease in rodent models by targeting the microbiota-gut-brain Axis. Front Aging Neurosci. 2017;9:403.

36. Zhang X, Dong H, Li N, Zhang S, Sun J, Zhang S, et al. Activated brain mast cells contribute to postoperative cognitive dysfunction by evoking microglia activation and neuronal apoptosis. J Neuroinflammation. 2016;13:127.

37. Jiang $P$, Ling $Q$, Liu H, Tu W. Intracisternal administration of an interleukin-6 receptor antagonist attenuates surgery-induced cognitive impairment by inhibition of neuroinflammatory responses in aged rats. Exp Ther Med. 2015;9:982-6.

38. Azizi G, Mirshafiey A. The potential role of proinflammatory and antiinflammatory cytokines in Alzheimer disease pathogenesis. Immunopharmacol Immunotoxicol. 2012;34:881-95.

39. Croker BA, Krebs DL, Zhang JG, Wormald S, Willson TA, Stanley EG, et al. SOCS3 negatively regulates IL-6 signaling in vivo. Nat Immunol. 2003;4:540-5.
40. Chhor V, Le Charpentier T, Lebon S, Ore MV, Celador IL, Josserand J, et al. Characterization of phenotype markers and neuronotoxic potential of polarised primary microglia in vitro. Brain Behav Immun. 2013;32:70-85.

41. Li Z, Liu F, Ma H, White PF, Yumul R, Jiang Y, et al. Age exacerbates surgeryinduced cognitive impairment and neuroinflammation in Sprague-Dawley rats: the role of IL-4. Brain Res. 2017;1665:65-73.

42. Desbonnet L, Garrett L, Clarke G, Kiely B, Cryan JF, Dinan TG. Effects of the probiotic Bifidobacterium infantis in the maternal separation model of depression. Neuroscience. 2010;170:1179-88.

43. Kobayashi $Y$, Sugahara H, Shimada K, Mitsuyama E, Kuhara T, Yasuoka A, et al. Therapeutic potential of Bifidobacterium breve strain A1 for preventing cognitive impairment in Alzheimer's disease. Sci Rep. 2017; 7:13510.

44. Gronier B, Savignac HM, Di Miceli M, Idriss SM, Tzortzis G, Anthony D, et al. Increased cortical neuronal responses to NMDA and improved attentional set-shifting performance in rats following prebiotic (B-GOS((R))) ingestion. Eur Neuropsychopharmacol. 2018;28:211-24.

45. Silk DB, Davis A, Vulevic J, Tzortzis G, Gibson GR. Clinical trial: the effects of a trans-galactooligosaccharide prebiotic on faecal microbiota and symptoms in irritable bowel syndrome. Aliment Pharmacol Ther. 2009;29:508-18.

46. Tzortzis G, Goulas AK, Gee JM, Gibson GR. A novel galactooligosaccharide mixture increases the bifidobacterial population numbers in a continuous in vitro fermentation system and in the proximal colonic contents of pigs in vivo. J Nutr. 2005;135:1726-31.

47. Jeong JJ, Woo JY, Kim KA, Han MJ, Kim DH. Lactobacillus pentosus var. plantarum C29 ameliorates age-dependent memory impairment in Fischer 344 rats. Lett Appl Microbiol. 2015;60:307-14.

48. Jung $\mathbb{H}_{\text {, Jung } M A}$, Kim EJ, Han MJ, Kim DH. Lactobacillus pentosus var. plantarum C29 protects scopolamine-induced memory deficit in mice. J Appl Microbiol. 2012;113:1498-506.

49. Beilharz JE, Kaakoush NO, Maniam J, Morris MJ. The effect of short-term exposure to energy-matched diets enriched in fat or sugar on memory, gut microbiota and markers of brain inflammation and plasticity. Brain Behav Immun. 2016;57:304-13.

50. Sun L, Dong R, Xu X, Yang X, Peng M. Activation of cannabinoid receptor type 2 attenuates surgery-induced cognitive impairment in mice through anti-inflammatory activity. J Neuroinflammation. 2017;14:138.

51. Terrando N, Monaco C, Ma D, Foxwell BM, Feldmann M, Maze M. Tumor necrosis factor-alpha triggers a cytokine cascade yielding postoperative cognitive decline. Proc Natl Acad Sci U S A. 2010;107:20518-22.

52. Cohen SJ, Stackman RW, Jr. Assessing rodent hippocampal involvement in the novel object recognition task. A review. Behav Brain Res 2015;285:105-117.

53. Hager AM, Dringenberg HC. Training-induced plasticity in the visual cortex of adult rats following visual discrimination learning. Learn Mem. 2010;17: 394-401.

54. Sears RM, Schiff HC, LeDoux JE. Molecular mechanisms of threat learning in the lateral nucleus of the amygdala. Prog Mol Biol Transl Sci. 2014;122:263-304.

\section{Ready to submit your research? Choose BMC and benefit from:}

- fast, convenient online submission

- thorough peer review by experienced researchers in your field

- rapid publication on acceptance

- support for research data, including large and complex data types

- gold Open Access which fosters wider collaboration and increased citations

- maximum visibility for your research: over $100 \mathrm{M}$ website views per year

At BMC, research is always in progress.

Learn more biomedcentral.com/submissions 